\title{
Situatedness:
}

\section{The Interplay between Context(s) and Situation ${ }^{1}$}

Katharina J. Rohlfing, Matthias Rehm, Karl Ulrich Goecke

Graduate Program Task Oriented Communication, Bielefeld University

Direct correspondence to Katharina Rohlfing, Applied Computer Science Group, Faculty of Technology, Bielefeld University, P.O.Box 1001 31, 33501 Bielefeld, Germany

Email address: kjr@uni-bielefeld.de (Katharina J.Rohlfing)

\begin{abstract}
In order to interpret the behaviour of cognitive systems, the integration into their specific cultural environment must be considered. The phenomenon of situatedness is a crucial determinant of this behaviour. We derive the notion of situatedness from the interplay between agent, situation, and context (divided into inter- and intracontext).

The main objective of this paper is to connect a theoretical analysis of situatedness with its implications for empirical research. In particular, we consider processes of situated learning in natural and artificial systems.
\end{abstract}

Key words: situated cognition, concept formation, learning, symbol grounding

\footnotetext{
${ }^{1}$ This work was carried out as part of the Graduate Program Task Oriented Communication (GK 256) which is supported by the German Research Foundation (DFG).
} 


\section{Motivation}

One of the difficulties connected to understanding and modelling natural systems is based on the fact that they are not isolated from but rather integrated into context and situation. However, if the goal is to observe mechanisms and processes of a system, it is methodically essential to detach the system from the situation it is part of. A growing sensitivity has emerged in theoretical and empirical research of cognitive science concerning this phenomenon which has been termed situatedness. It has been employed by sociologists, anthropologists, cultural psychologists, and conversation analysts as well as AI and behavioural scientists in describing the close relation between an individual and its environment, and the influence of this relation on the nature and development of the individual itself.

This paper is motivated by our aim to connect a theoretical analysis of the role of context(s) and situation with two examples of learning in artificial and natural systems. We will point out some aspects of the interplay between context and situation in order to contribute to the scientific exchange regarding situatedness in the field of (linguistic) concept formation. We start by clarifying the notions context and situation involved in the phenomenon (s. section 2). Then, some theoretical underpinnings for the application of these notions to acting systems are outlined (s. section 3). In sections 4 and 5, we report from two different perspectives on which impact situatedness has on human cognition or more generally, processing of information in an acting system.

First, an artificial system is described that models processes of situated learning (section 4). Afterwards, language acquisition in humans is viewed from a situated perspective (section 5). Finally, we summarize our analyses and propose a definition of the notion situatedness (s. section 6). 


\section{Context vs. Situation}

\subsection{Terminology}

The terms situation and context are often used synonymously. However, to derive situatedness from the interaction of situation and context it is necessary to dissociate these notions.

A situation consists of the spatiotemporal ordering of objects and agents alongside physically given constraints or characteristics like gravitational force or light intensity. Of interest to an agent $^{2}$ are the stimuli that it can perceive with its sensors. In our view, situatedness refers to specific situations in which actions take place. Actions are understood here not only as taskoriented behaviour but in a broader sense (cf. Clancey, 2002). In contrast to situation, context is a general construct that depends on various factors and is definable on at least two different levels. On the one hand, there are socio-cultural (global) contexts such as language. On the other hand, smaller (local) contexts can also be found, e.g., the context of a seminar. The actions of an individual, from now on called an agent, are constrained by this context. A student at a seminar has to act according to her role as a student, i.e. she has to be attentive, ask smart questions, and discuss the topic of the seminar. Dancing, singing, or swearing is not expected from her as it is not licensed by the seminar context. In this sense, a context supplies certain patterns of behaviour and of analysis for situations an agent can be confronted with. A provision of this kind is exemplified in section 5. A situation is thus embedded in a certain context. This context influences or determines a situation and its analysis by the agent. In a given situation, there is not a single context but a great number of different, possibly overlapping contexts. In order to analyze whether something is context sensitive or situated,

\footnotetext{
${ }^{2}$ When we talk about agents, we mean not only human agents but also artificial systems, following a definition given by Franklin \& Graesser (1996): "An autonomous agent is a system situated within and a part of an environment that senses that environment and acts on it, over time, in pursuit of its own agenda and so as to effect what it senses in the future."
} 
it is necessary to look at the specific situation and figure out which contexts are present and which role the agent plays in each of them.

We will now approach context in a more abstract way. For this purpose we divide it into two different types: intercontext and intracontext. Both of them are established by agents. Clancey (1993) describes the interactions of a group of agents as follows: "In a group we are mutually constraining each other's perception and sequencing, so our capabilities to interact are both developed within, and manifestations of, social, multiagent interactions." When agents interact with their environment or with one another, they establish what will here be called an intercontext. Such an intercontext is not an objective fact, but it consists of a complex network of interacting subsystems (society, study group, etc.). Semin \& Smith (2002) call this product of agents' interactions the "socially shared reality." Thus, an intercontext is a social phenomenon that cannot be surveyed by a single agent, rather it hints at how an agent can interact appropriately in a specific situation, i.e. the agent's options for acting. Further it suggests probable ways of how an agent acts and gives meaning to the situation. By its interactions in specific situations in a certain intercontext, an agent establishes its own intracontext, allowing it to make sense of the situations it encounters, i.e. to give meaning to them. The intracontext is a partial mapping from the intercontext.

There is a mutual influence between these two types of context. The intercontext influences the intracontext, because the intracontext of an agent is created in specific situations where the intercontext as socially shared reality manifests itself. There is also an influence the other way around: the agent makes sense of the specific situation and acts in it on the basis of its intracontext. The action itself, in turn, is part of the intercontext. We will disregard this interdependence here to make the analysis of contexts and situatedness feasible. 


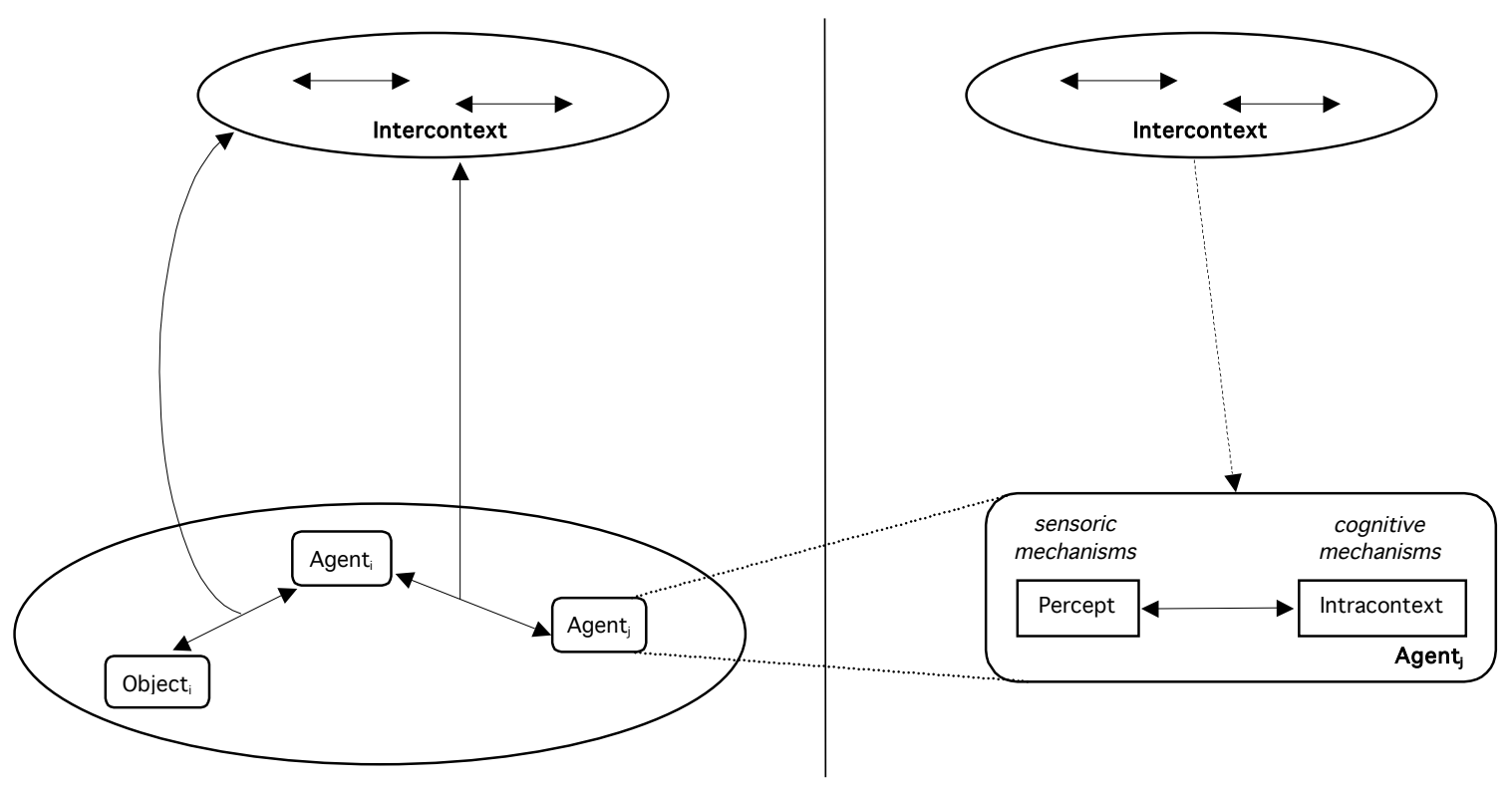

Figure 1: Inter- vs. Intracontext. Agents interact with each other and with objects. These interactions establish the intercontext (left hand side). Simultaneously, an individual agent builds up an intracontext, which is a partial mapping from the intercontext (right hand side).

Against the background of the terminology developed here, establishing an intracontext, i.e. abstracting away from specific situations, is learning. A single learning step is situated as it takes place in a specific situation. It is influenced by the intercontext in which the situation is embedded, supplying certain interpretations and action patterns. The result, i.e. the abstraction over various (similar) situations, is thus (inter)context sensitive. The established intracontext is always based on the intercontext encountered during learning. Using the intracontext in a specific situation is the characteristic feature of situatedness.

\subsection{Situated action and situated learning}

An action is situated because it always takes place in a specific situation. The more sophisticated an agent's intracontext, the more sophisticated actions become possible by taking more of the situational setting into account. An example of an action that is mostly 
rule-driven and utilises only a limited fraction of the specific situation can be found in Franklin (1995). A certain type of wasp needs a paralysed cricket to feed the wasp grubs. The wasp brings the cricket to the threshold of the burrow in which it will lay the eggs, goes inside to check the burrow and then pulls the cricket in. If the cricket is removed a few centimeters from the threshold before the wasp reappears, it comes out, brings the cricket to the threshold, goes inside to check the burrow, etc. This can be repeated over and over again. The wasp is following a genetic plan, triggered by cues present in the situation. Actions are selected on the basis of a pre-existing schedule and these triggers. In this case, no elaborated intracontext is mediating the action selection process.

Learning, viewed as a process of concept formation, constitutes the other end of the scale of situated processes. Over time, an agent collects relevant information from specific situations and forms concepts. What is a relevant piece of information in a situation is influenced by the intercontext, since the same situation given in different intercontexts provides different relevant pieces of information for an agent. These concepts constitute the intracontext of the agent. Sections 4 and 5 discuss this issue in detail.

\section{Interaction Mechanisms}

In an acting system, two major mechanims allowing the interaction of the agent with its environment can be distinguished. They are termed perceptual and effectorial processes. All mechanisms constituting the sensoric perception of the environment by the agent (sensoric mechanisms) as well as their interpretation on a "higher," deliberative level carried out by cognitive mechanisms are perceptual. On the other hand, the notion of an effectorial process comprises all cognitive and motoric subprocesses controlling the actuators of the agent. In this way, the agent actively exerts an influence on the situation. 
Situatedness can here be interpreted as follows: the agent interacts with the situation by perceptual and effectorial processes. The intracontext is formed by concepts permanently modified by the interaction (see section 4 and 5). This interplay of intracontext, perceptual, and effectorial processes on the one hand and the situation on the other hand is the characteristic feature of situatedness, which is most obvious in simple stimulus-response couplings, e.g., reflexes. These very basic interaction mechanisms form one end of a scale denoting a "measure of situatedness" of actions. At the other end are actions in which perceptual and motoric processes play only a secondary role regarding the interaction between agent and situation. For about two decades, the above mentioned interaction mechanisms have been the focus of research activities. Two areas are of special interest here: the discussions concerning the notions of a) symbol grounding and b) situated action execution. Symbol grounding is concerned with representational levels, structures, and processes occuring in and constituting interaction mechanisms. Situated action execution deals with the question of how far interaction mechanisms can be described by methods of classical AI (search, planning, knowledge representation using logic-based formalisms etc.) or of whether a new paradigm with respect to agent architectures is needed (cf. Greeno et al., 1993).

\subsection{Symbol Grounding}

Symbol grounding denotes the issue that, besides its syntactic role within a system, a symbol has an additional meaning constituted by means of reference to (parts of) the environment (see Harnad, 1990). Thereby, an agent is not limited to using the symbol as if it were solipsistic. Due to the principle of compositionality, the agent also knows the meaning of complex symbols derived by mere syntactic manipulation of grounded symbols. With respect to the aforementioned interaction mechanisms, symbol grounding has mainly been dealt with 
from the perceptual perspective. This seems due to the tradition of AI research where artificial systems are mostly conceptioned as 'recipients of orders' not supposed to carry out complex interactions with the situation, i.e. their effectorial tasks are very limited (e.g. data base systems). However, an agent, not only perceiving the situation but also acting in it, has to be able to map its intracontextual symbols onto motoric patterns. We are aware of the controversial debate about whether agents use symbols (Madole \& Oakes, 1999; Keijzer, 2002). From our perspective the notion of intracontext comprises both perceptual and conceptual processes in agents, irrespective of whether symbols play a role in these processes or not.

Symbol grounding is crucial for the discussion of the notion of situatedness if one accepts the hypotheses that a) there exists a regular relationship between the symbols and the context, and that b) complex agents possess a level where their behaviour is symbolically expressable and that this influences their behaviour in fundamental ways. The interaction between agent and environment is only possible in an architecture allowing for the formulation of solutions of the symbol grounding problem. For an agent, the physical features of a situation (objects, other agents, etc.) are stimuli generating a subsymbolic representation within the agent (s. figure 1 (right hand side)). For natural agents (e.g. humans), this is the activation of neurons in the sense organs. In artificial agents, it can be, for example, pixel images, the activation of contact sensors, or the diaphragm of a microphone. From this sensoric representation, the intracontext is established (e.g., symbols are derived, Goecke \& Milde, 1998), possibly including additional, subsymbolic layers (e.g. neural networks). On the other hand, the intracontext propagates onto a subsymbolic level, this time the motoric control level. In this way, the agent affects the situation. It is still unclear to what extent perceptual and effectorial processes use different representations (Neumann \& Prinz, 1990). 


\subsection{Situated Action Execution}

The notion of situated action execution has achieved a wider acknowledgement in the context of the appearance of the behaviour-oriented paradigm of action control (e.g. Brooks, 1991). Earlier "classical" systems have been designed according to the so-called Sense-ModelThink-Act (SMTA) architecture: the perception of the environment leads to the construction or modification of a world model. A planning algorithm computes a sequence of actions to be performed according to which the control parameters of the actuators are modified (Nilsson, 1984). The main problem with this kind of architecture is the temporal delay of (possibly important) reactions to changes in the environment due to the necessity for complex symbol manipulation (deliberation, e.g. inferencing or replanning) before motoric processes can be started. In the framework of the behaviour-oriented paradigm, situated action execution means that the agent does not act on the basis of a fixed action plan (cf. also Clancey, 2002), but (at least additionally) on the grounds of specialised sensor-effector interconnections allowing for fast reactions in a changing environment. No explicit symbolic representations are needed for this kind of reactive behaviour. Quite to the contrary: as symbols are generally not used in this kind of action control, they have to be derived from distributed pieces of information (see previous section) if they are to serve as a descriptive level of deliberative processes.

Examples of agent architectures designed after these principles comprise Brooks' mobile robots (cf. Brooks, 1986) employing the so-called subsumption architecture, and Steels' dynamical systems approach (Steels, 1993). Typically, these agents do better than SMTA agents with respect to speed and simplicity of modelling, especially in dynamically changing environments where no long-term goals have to be pursued.

An attempt to connect the advantages of both control architectures and thus to compensate for the respective disadvantages are hybrid systems (Malcolm \& Smithers, 1990; Milde et al., 
1997). Here, reactive pieces of behaviour are influenced by control parameters brought about by processes on the cognitive level. In this way, the intracontext of an agent modifies, if necessary, the situationally determined action selection. More recent approaches enhance these types of systems with the possibility to consider also intercontextual influences. Dautenhahn et al. (2002) calls them socially situated agents: "A socially situated agent acquires information about the social, as well as physical domain through its surrounding environment, and its interactions with the environment may include the physical as well as social world." The next section shows an example of an agent using language as a social influence.

\section{LOCATOR: Modelling concept formation in situated agents}

In the previous sections we have developed the notions of inter- and intracontext and have discussed how symbol grounding and situated action execution contribute to a situated agent architecture. On this basis we will exemplify how an intracontext is established by forming concepts.

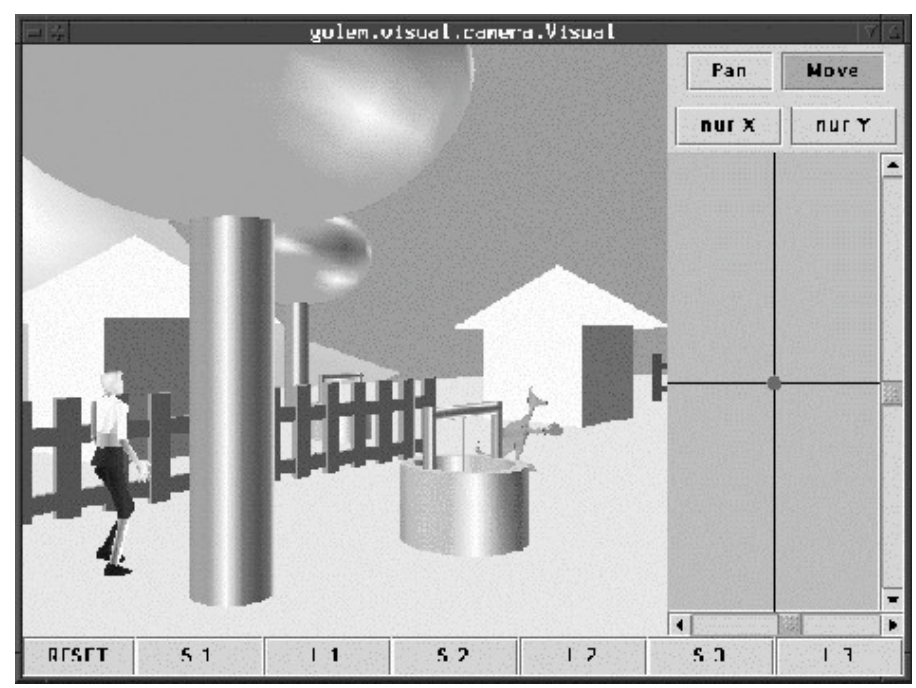

Figure 2: LOCATOR: an anthropomorphic agent explores its complex, virtual environment. For the agent, this situation is constituted of the visual perception of the scene and the language input, here e.g., The well is right of the fence. 
Concepts are understood here as bridging the gap between perceptual experiences and linguistic symbols. Thus they serve two functions. On the one hand, they ground linguistic symbols in sensorimotor experiences. On the other hand, they help structuring sensorimotor experiences by an abstract amodal symbol system. A concept in LOCATOR can be seen as the organisational unit that binds certain parts of sensorimotor acitivity to linguistic symbols. The literature on concepts is very rich and diverse (see Margolis \& Laurence, 1999, for an overview). The notion of concept as used in LOCATOR can best be described as a dynamic approach to concepts (see, e.g., Lakoff, 1987; Thelen \& Smith, 1994). Such an approach denies the objectivist's perspective on concepts and propagates instead a subjective process of concept formation, i.e. dependent on experienced situations and tasks. By this change in perspective the focus of investigation is shifted from a concept's content to the process of concept formation. Stability in the use of concepts thus stems from universal concept formation mechanisms and a stable environment. This view corresponds to Glenberg's ideas on the variability of cognitive structures, which is limited because of "our common human endowments and our common environment" (Glenberg, 1997). This does not necessarily imply identical concepts in different agents. What's dynamic in dynamic concepts is their ability to remain inherently changeable during the lifetime of an individual.

LOCATOR is a simulation system that is well suited to examine aspects of situated cognition like concept formation. ${ }^{3}$ Virtual, anthropomorphic agents move around in a virtual, complex world (see figure 2). The agents autonomously explore their environment. They are controlled by a behaviour-based architecture. Different sensors allow them to receive two kinds of input during their exploration: vision and language. Concepts are formed in the specific situations an agent finds himself in during this exploration. Thus the concepts reflect the agent's history of experience. Situations vary across agents due to their different

\footnotetext{
${ }^{3}$ LOCATOR is based on the system LOCUTOR, which was developed by Jan Torsten Milde at Bielefeld University (e.g. Milde, 2000).
} 
exploration histories, which depend on the agent's exploration path and on the language input which is supplied by the human interlocutor.

The domain chosen for LOCATOR are frames of spatial reference as described by Levison and his colleagues (see e.g. Bowerman \& Levinson, 2001; Levinson, 1996; Senft, 1997). The language input describes spatial relations between the objects the agent encounters. It is given in one of two languages: either in German (relative frame of reference) or in Marquesan ${ }^{4}$ (absolute frame of reference). The effects of situated concept formation can be found on two different levels in LOCATOR:

1) Comparing agents experiencing different languages: Marquesan structures the spatial domain in a way that is compatible with a kind of absolute frame of reference. The language input given in German employs a relative frame of reference. Thus, by comparing the established concepts, the effects of the different intercontexts can be shown.

2) Comparing agents experiencing the same language: The effects of situated concept formation lead to concepts that differ in content, although the agents experienced the same language. Each agent establishes an individual intracontext.

The language input plays a crucial role in LOCATOR. In conjunction with the objects and the agent itself it establishes the intercontext of the agent. The intercontext exerts an influence on the learning process, especially by means of the language input. The language input is a conventionalised form of the intercontext and thus more than a situated input component. It represents a generally accepted way how a certain language community may describe the spatial arrangement of objects in a given situation. During the learning phase, language is used by the agent as a tool to analyse situations, e.g., to focus on relevant elements of the scene. On the one hand, language is functionalised as a selection criterion. On the other hand,

\footnotetext{
${ }^{4}$ Marquesan is a polynesian language applying primarily an absolute frame of reference with a directed axis seaward/landward, and an undirected crossaxis. We are grateful to Gabriele Cablitz (MPI Nijmegen) for supplying us with the utterances used in LOCATOR (see Cablitz, 2002 for further details).
} 
utterances are directly grounded in the sensoric input which is an additional basis for concept formation. A concept is formed by abstracting from different situations and an intracontext consists of these concepts.
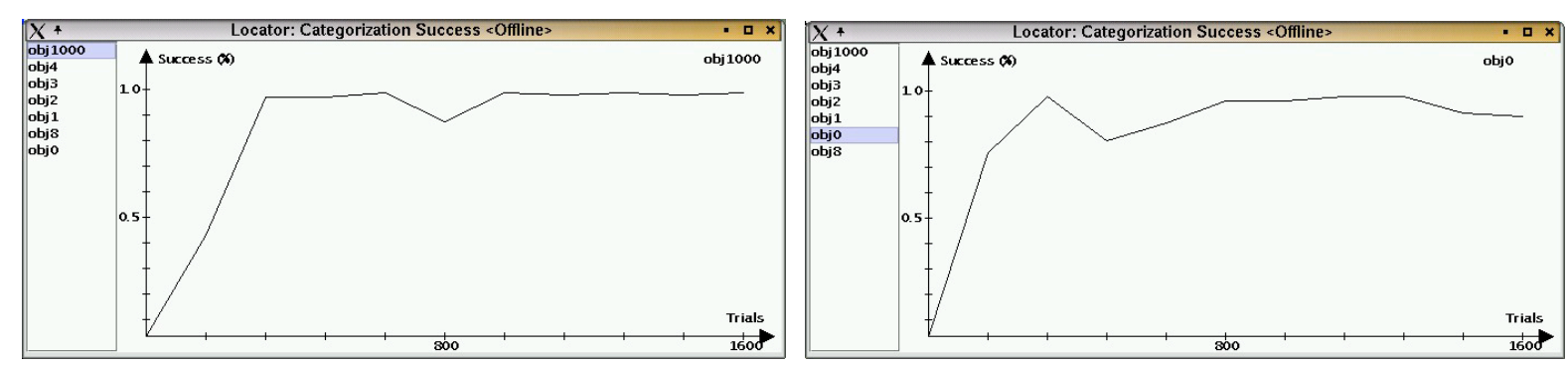

Figure 3: The development of the categorisation success rate of two agents over time. Agent one (left hand side) experienced German language input, agent two language input from Marquesan.

One important result of LOCATOR is the dependence of concept formation on specific situations. The intracontext established by concepts depends on the experienced situations. On the basis of these experiences, an abstraction is possible allowing for the categorisation of different situations as similar.

This needs focusing on features relevant for the categorisation process and for constituting the basis for the formation process. In different agents, different features may emerge according to the situations they are confronted with. This is in accordance with findings of Schyns \& Rodet (1997), who show that the features used by subjects to categorise objects are created in a situated fashion and depend on the subject's individual history of experience with the objects.

Some of the results can be described here (for a more detailed discussion see Rehm (2001a and 2001b). Figure 3 shows the categorization success for two agents. Agent 1 on the left hand side was provided with language input in German. In this experiment only spatial descriptions employing the relations links (left) and rechts (right) were used. The language input of agent 2 on the right hand side was given in Marquesan. Here, the relations ma tai $o$ (seaward) and ma uta o (landward) were used. What is shown is the success rate of the two 
agents on a scale from $0(0 \%)$ to $1.0(100 \%)$. Every 160 trials the average success is measured over this time period. In a single trial, an agent receives - apart from the permanently available visual input - a spatial description by the human interlocutor. The agent tries to categorize the input with the already established concepts. If this does not succeed, concepts are created, modified or the perceptual system is modified. Both agents have established concepts that allow a successful categorization of incoming input, as figure 3 shows. During the process of concept formation the agents shape their abilities to analyse the perceptual input. First the dimensionality of the three-dimensional input image is reduced by calculating the center of mass of each object. Then, five perceptual features are calculated by each agent. Three of them rely on the main axes of human spatial cognition (Howard, 1982): the vertical, the 2. sagittal (depth), and the horizontal. The features calculated are:

1) the angle between the center of mass vectors (COM) of two objects and the vertical (VERTIKALE);

2) the angle between the COM of two objects and the horizontal (HORIZONTALE);

3) the angle between the COM of two objects and the second sagittal (2. SAGITTALE);

4) the distance between two objects (ABSTAND);

5) the alignment of two objects in reference to the origin of the coordinate system

\section{(AUSRICHTUNG).}

Figure 4 depicts the perceptual systems of the two agents. The agents establish discrimination nets for a number of perceptual features. This technique was first introduced by Steels (e.g., Steels, 1996). Both agents have modified their systems in a way that allows a successful categorization, i.e. one that is compatible with all experienced input (vision and language). A single node of such a discrimination net can serve as a feature of a concept. The activation of a node thus also leads to some activation in the associated concepts. Comparing the two agents shows two different effects the variation of the language input has. The two agents 
establish different coordinate systems in which the perceptual features are calculated (highlighted on the left hand side of figures 3 and 4). Agent 1 (relative frame of reference) uses a coordinate system centred in itself (obj 1000). Agent 2 (absolute frame of reference) calculates the features in a coordinate system centred in the mountain top $(\operatorname{obj} 0)$. The other effect can be seen directly in figure 4 . The agents have modified their respective perceptual system in different ways, focusing on different features to establish spatial concepts.
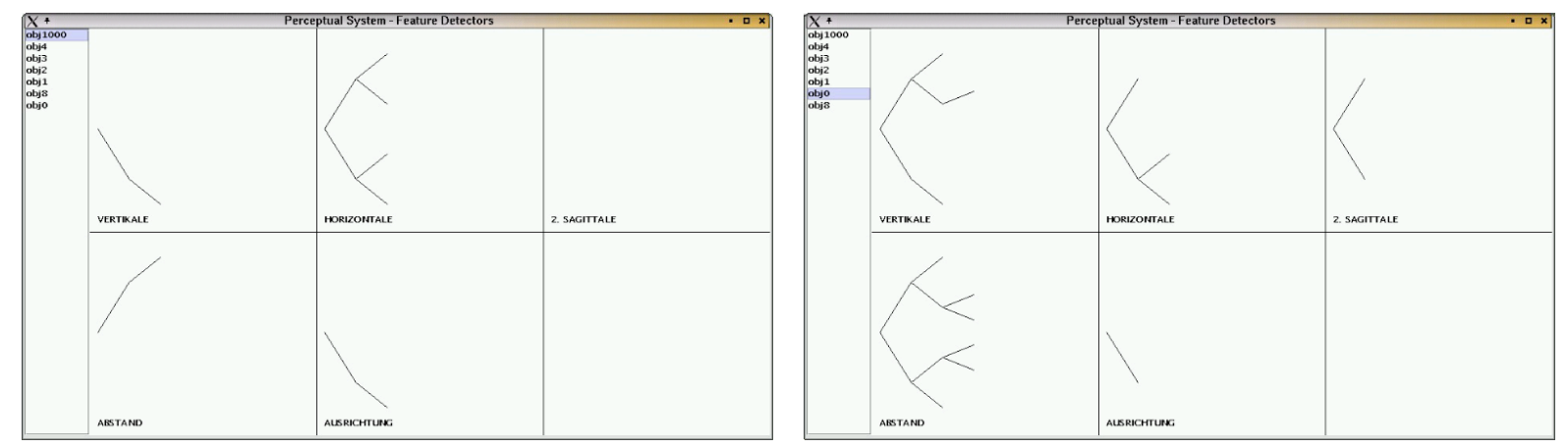

Figure 4: Features created by the two agents. Agent one (above): German. Agent two (below): Marquesan.

Different feature detectors were elaborated by the two different agents. Moreover, suitable anchor points for the calculation of perceptual features have emerged as can be seen on the left hand side: for agent one obj1000 (the agent itself) is highlighted, for agent two it is obj0 (the mountain top).

With regard to the notions developed in this article, the system LOCATOR exemplifies two important aspects. As the nodes of the discrimination nets serve as building blocks for the established concepts, the content of these varies accordingly. The features they created and thus the concepts formed on the basis of these features are affected by the different situations the agents experienced. Note that the agents were embedded in different intercontexts. The intracontext is also subject to this variation because it consists of the concepts formed and thus it is agent-specific. 


\section{Situated Learning}

In the previous section we claimed that concept formation is the process of establishing an intracontext. In this section, we apply these considerations to human development. Generally, we follow Vygotsky's (1987) idea, who claims that concept formation in humans is not a gradual socialization introduced from the outside, but a gradual individualization that emerges on the foundation of the child's internal socialization. We focus on language acquisition and establishment of the meaning of a spatial preposition, which involves questions about how a representation is grounded. The following psycholinguistic study exemplifies some relevant problems. In this study, the infants' understanding of local prepositions in Polish was tested (Rohlfing, 2001).

\subsection{The acquisition of local prepositions}

The meaning of a local preposition is usually captured by the appropriate spatial relation (cf. Herweg, 1989). Accordingly, the child has to grasp the abstract or lexical meaning of this relation first when acquiring the local preposition. However, Bowerman and Choi (2001) review studies indicating that children make use of their intracontextual prelinguistic knowledge about objects and events. Additionally to their linguistic knowledge, infants follow certain strategies of which some will be presented in this section and discussed from the situated point of view.

The notion of strategy originates from Clark's (1973) discussion of the results from her study of infants' understanding of spatial relations. She observes that infants pay attention to the features of objects: if a presented object includes a surface, the infants' behaviour is influenced insofar as they will declare it the landmark-object and put something on its surface. If the presented object is a container, they will put other objects IN this landmark. This type of influenced behaviour dominates over the language understanding, i.e. infants 
will put e.g. the pot $\mathrm{ON}$ the table even though they are verbally instructed to put the pot UNDER the table. Sinha (1982) observes a further strategy. Infants learn a canonical orientation of an object first, i.e. they learn how to place a cup on a table (i.e. in an up-right position), how to hold a pen, etc. This canonicality constitutes the basis of infants' knowledge about objects, their intracontext, and sets up conditions to recognize the object and to handle it correctly. This non-linguistic knowledge about objects already contains culture-specific aspects. How an object is handled as an artefact is grounded in canonical rules preserved by the cultural group. Cups, for example, do not occur naturally, but must be produced. To be a drinking vessel, a cup must be a container, which possesses a certain structure (Sinha, 1983). Canonical rules restrict this structure and we refer to a cup in its canonical position as a container. The motivation for canonical rules, however, depends on human interest and values. In our cultural group, a basket resides in its canonical orientation when it serves as a container for transporting something with the opening upright - and not with the opening upside down for to catch chicken as in the Zapotec culture (Sinha \& Jensen de López, 2000). Canonicality is grounded in children's experiences with objects and events and it builds up a child's expectations about these objects and events. In this sense, canonicality belongs to the intracontext of a child. However, the strategy to act according to the canonical rules develops when perceiving situations which are influenced by the intercontext, i.e. a child can build up a related concept when she or he is confronted with it in a certain situation and identifies the character of an object. Evidence for using the non-linguistic strategies is provided by the following study with 20-26 months old infants in which responses to instructions with spatial prepositions were evoked. The rationale of the study was to test the involvement of nonlinguistic knowledge and to get insight into its nature (see Rohlfing, 2001 for details). 


\subsection{Experimental Study}

The study we refer to follows a certain experimental setting: at the beginning of a task two objects were presented to every child. Subsequently, the infants were asked to put the objects together in a relation. Relation is meant as an alignment between two objects, the trajectory and the landmark. The terms originate from Langacker's approach (1987), according to which the trajector refers to an active object acting against the background of another object, the landmark. The alignment specified by the task requires a motion from the trajector to the landmark. Consequently, solving the problem and achieving a spatial relation in the task, the child decides:

- which of the objects is the trajector, which is the landmark?

- which relation is appropriate to the objects or which is suggested by them?

- which motion should be performed to achieve the relation?

In the following, trajector and landmark are designated also as roles of objects. The role of an object is specified by its function in an action. The action, in turn, is grounded in a situation.

\section{Method and Hypotheses}

In this study, infants were requested to bring two objects together in an ON-relation. Two types of verbal instructions 6 were used for this purpose: one type consists of a syntactically correct request (1) relating two objects to each other in view of an ON spatial relation; the other also consists of a request, but in this case this is syntactically incorrect (2): the preposition is omitted:

(1) Daj konika NA most! [Put the horse ON the bridge!]

(2) Daj konika most! [Put the horse the bridge!]

Assuming strictly situated problem solving, infants were supposed to perform well in both cases without indications of missing the preposition, because the solution was assumed to 
emerge from a child's knowledge of the objects. Additionally, only objects where the ONrelation was possible (e.g. a pot $\mathrm{ON}$ a table) were used in the task. For the analysis, the infants' reactions to the two types of instructions were compared.

\section{Results}

24 infants of the age of 20 to 26 months participated in this study. 83 responses were relevant for the scoring. A response was classified as non-relevant when the participant performed the requested relation with a different toy or did not react to the instruction at all. The scored reactions show a clear result: there are 41 responses to the instruction without preposition in which infants performed the ON-relation - as predicted -, only one child performed another relation. To the syntactically correct instruction with an ON-preposition, there were 38 responses with an $\mathrm{ON}$ - and three with another relation. The results support the hypothesis that infants do not need the appropriate preposition to understand an instruction when the relation requiring a certain situation allows for the most frequently seen roles of the focussed objects. Infants' reactions to the required task show that they are guided by certain strategies additional to their linguistic knowledge. The qualitative results of the experiment (s. case studies reported in detail in Rohlfing 2002) indicate that this strategic knowledge is important for infants in the age group studied. They are anxious to maintain it with the aid of games (in which they imagine and create already-seen situations) or imitations (in which they test similar situations). In this sense, frequency plays a role for correct understanding of language. However, frequency as a factor in understanding language cannot be seen as a determinant: according to the results reported in Sinha et al. (1999), frequency can influence but not substantially determine the semantic content.

Taken together, the results from the study reported here confirm the involvement of nonlinguistic strategies in understanding instructions. However, the way how the strategies 
influence infants' behaviour differs from the way suggested in Clark (1973) or Sinha (1982). In this sense, the results suggest a more complicated picture about the involvement and the nature of infants' non-linguistic knowledge. While both authors use the term rules to characterize the influence of non-linguistic strategies, a case study from the above experiment shall cast doubt on this claim. It questions whether these strategies determine (in the case of rules) infants' behaviour or just influence it, leaving open the possibility that other factors contribute to the behaviour.

Marcel (23 months old) received the instruction to put the cup plate and he put the plate ON the cup. The expected reaction, however, was an inverted one: the cup ON the plate, because the plate has a surface (Clark's rule) and because the child knows that usually things are put on the plate (canonicality by Sinha). After consultation with his mother it turned out that she always makes tea that way: she puts the tea in a cup and then covers it up with a plate to stew. In this situation, both objects seem to be known to Marcel, whereby the plate received a trajector-role and was put $\mathrm{ON}$ the cup. In this case, the object's role is deduced from its "relational character" (cf. Thiel, 1985) and influenced by the coexistence of another object. Marcel matched both objects to a typical, for him well-known situation. In that situation, a certain activity (making tea) involves both objects and ends with a certain relation - to put the plate $\mathrm{ON}$ the cup. Later during the experimental session, Marcel demonstrated that when other objects were present and he was focussing on either the cup or the plate, he preferred the canonical positions (the plate to put things ON it and the cup upright to put things IN it). Thus, one cannot claim in this case that only physical properties of the objects provoke a certain behaviour of a child and that the child's knowledge about objects consists only of their canonical relations. The child's response results from the interplay between her or his intraand intercontext in a specific situation. 


\section{Discussion}

The results show that infants do not need prepositions for following an instruction if the relation requested in this instruction correlates with the most-seen role of an object. Beyond it, Marcel's reaction conveys an idea of how situated learning of a relation can proceed: the child perceives a certain situation in which two objects are involved in an activity. From this performance a child can deduce the individual roles (trajector or landmark) of involved objects. The typicality of a situation (Rohlfing, 2001) therefore seems to be central to the child's intracontext: the experience of a situation provides information, which is substantial for a child building up her or his knowledge about involved objects. In a further, similar situation the child associates a known action with these objects and regards their canonical orientation. The child extends her or his knowledge about an object observing its roles depending on different activities in different situations. Information from these observations creates a character of an object - a kind of concept.

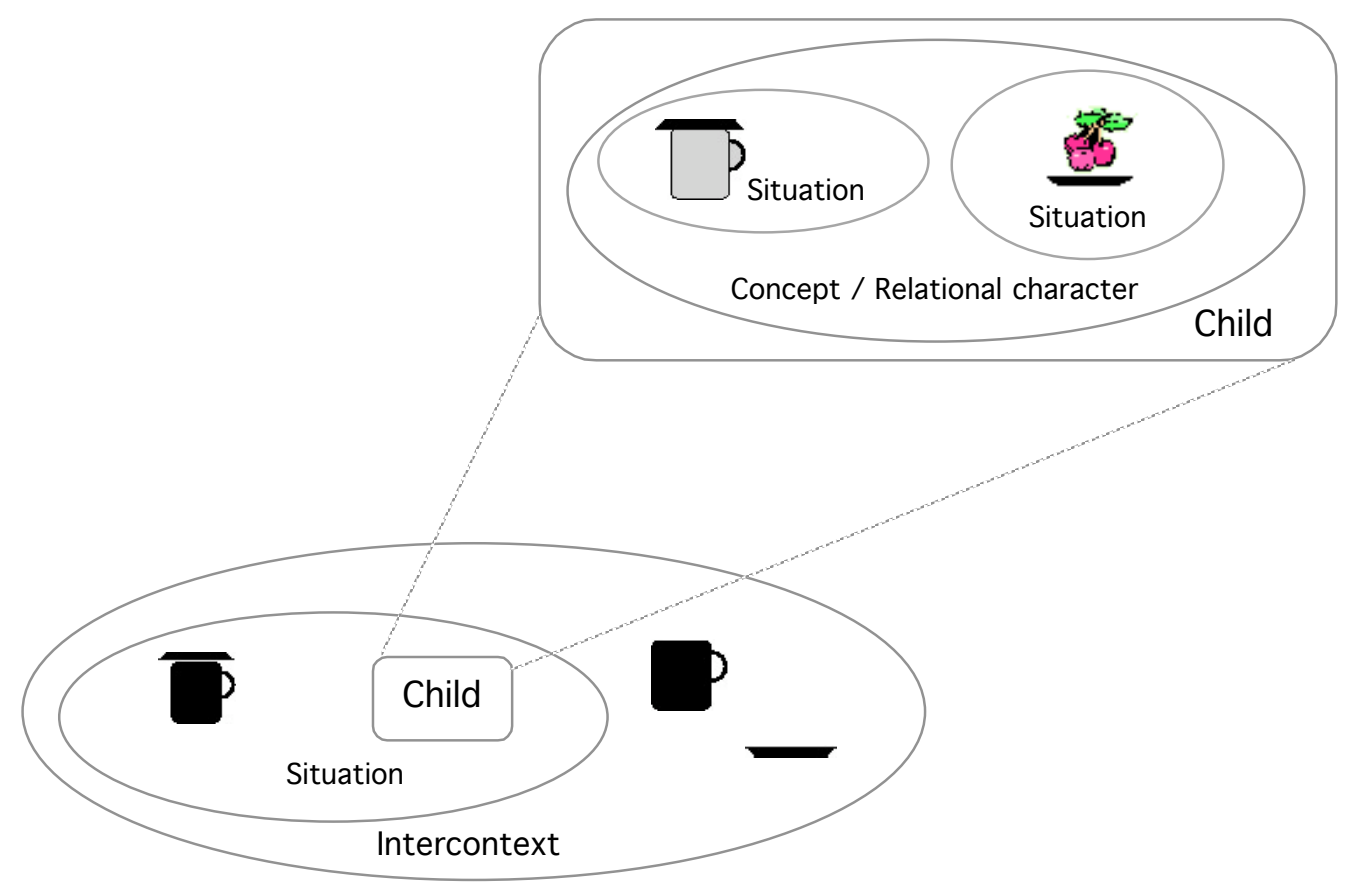

Figure 5: Forming an intracontext from an intercontext. 
This mechanism of creating a meaning is also suggested in Riegler (2002), when he points out that "[m]eaning arises as a result of relating a new piece of experience to the existing network of already made experiences rather than to entities in the world." On this basis, the child knows which relations are possible with this certain object (see figure 5).

To put it in other words, the character of an object consists of the sum of its functional roles: if a plate functions only as a support (e.g. for food), the child will know the plate only in this role. In a recurring situation, seeing a plate, the child activates this role and tries to relate other objects to it appropriately (e.g., the child will put a toy horse ON the plate). This intracontextual knowledge dominates the child's upcoming action in a situation and bears an important function: the child knows, for example, what a plate is for. If she or he had already observed other roles of an object, it depends on a given situation and involvement of further objects which role from the objects' 'character repertoire' will be motivated. When, in turn, a situation is designed to motivate a certain relation - as in the presented study - it is possible that infants understand an instruction without a preposition i.e. without marking the spatial relation verbally as well as an instruction with an appropriate preposition. In this case, the understanding is supported by the situation: the child's intracontext responds to a certain situation, whereby the perception motivates a non-linguistic strategy. Grounding in an object's character (i.e., the state of knowledge about an object), a strategy allocates a certain role to an object.

Marcel's reaction provides counterevidence to the mechanisms of non-linguistic knowledge presented in Clark and Sinha. Learning proceeds in a situated fashion and is not subject to only one rule (e.g., canonicality) but to different strategies being applied to specific situations. Moreover, the strategies which represent an action originating from the intracontext are established on the basis of a situation and information from the intercontext. 


\section{Summary}

We have subdivided the notion of context into intra- and intercontext. An agent's intracontext is constituted of concepts formed and modified during situated perception-action cycles. The intercontext of a group of agents emerges from the interplay of the members of this group with the environment and each other. As a social phenomenon, it restricts the interactions of an agent in a specific situation. As we have shown by our examples, this distiction between an inter- and an intracontext is essential. Even though other authors do not make it explicit, they acknowledge that cognition is "distributed, occurring not only within an individual mind [intracontext] but extended across other people or elements of the environment [intercontext]" (Semin \& Smith, 2002). The dovetailing of an agent's intracontext and the situation, mediated by perceptual and effectorial processes, is the essence of situatedness. This is a much broader sense of situatedness than is usually found, e.g. in Dautenhahn et al. (2002). When they speak of situatedness, they mean just acting in a specific situation. The interplay between inter- and intracontext, i.e. the strong structural coupling of an agent with its (phyical and social) environment is termed by the authors embeddedness. In contrast to their terminology, we propose that many phenomena - like embeddedness or the interpersonal variation in conceptualisation of a situation (Fischer, 2000) - can be explained by the two different types of context and their interplay. In AI-oriented cognitive science, situatedness can be found especially in two fields: symbol grounding and action selection. At least partially, symbols used intracontextually have to be reflected on correlates in a situation to be able to acquire meaning. Research questions connected to symbol grounding are: What kind of representation allows this connection? What kind of processes lead to such representations? Concerning action selection, a major issue is how often the perception-action cycle is run through in a certain time span and to what extent complex intracontextual representations are necessary for adequate (re-)actions 
of the agent. Here, the poles are classical planning and reactive systems, respectively. Changing perspective from symbol grounding and action selection to the establishment of an agent's intracontext emphasises the importance of situatedness. Building up an intracontext always takes place in a situation and thus always refers to the situations an agent encounters during its interactions. The intercontext supplies patterns of analysis, e.g. in the conventionalised form of language. Therefore, language is more than a situated input component. The specific form of the intracontext depends on two things: the situations encountered during learning and the agent's intercontext. By means of the system LOCATOR and the case study of Marcel in comparison to other children, it was shown how a situated learning process can lead to differences in the conceptualisations of different agents despite equal success in using their concepts. Our main focus is on the process responsible for establishing concepts and thus the intracontext. On the one hand, this clarifies the influence of the intracontext on the agent's interactions and on the other hand, it emphasises the dynamics of intracontextual change. In our approaches we focused on situated learning because it is always part of an interaction, i.e. interaction cannot take place without situated learning. Three types have to be distinguished: concept formation, modification, and preservation. Concept formation is the process of initially building up the intracontext on the basis of information from the intercontext given in specific situations. Intercontextual change and experience of new situations have to be accompanied by changes in concepts. As concepts constitute the intracontext and are responsible for an appropriate action, it is necessary to modify them and to keep them updated. As the agent's intracontext interacts with the environment, every action is a preservation of already existing concepts; the successful action confirms the character of these concepts.

We summarise: an agent interacts with the situation by perceptual and effectorial processes. On this basis concepts are formed and permanently modified by the interaction. The 
intracontext is established by the formed concepts. This interplay of intracontext, perceptual and effectorial processes, and the situation is the characteristic feature of situatedness. Thus, situatedness is the effect of a situation on concept formation (situated learning) and the concrete manifestation of an action in a situation.

\section{References}

Bowerman, M. \& Choi, S. (2001). Shaping meanings for language: universal and languagespecific in the acquisition of spatial semantic categories. In M. Bowerman \& S. C. Levinson (Eds.), Language acquisition and conceptual development, (pp 475-511). Cambridge University Press, Cambridge.

Bowerman, M. \& Levinson, S. C. (Eds.) (2001). Language acquisition and conceptual development. Cambridge University Press, Cambridge.

Brooks, R. A. (1986). A robust layered control system for a mobile robot. IEEE Journal of Robotics and Automation 2, 14-23.

Brooks, R. A. (1991). Intelligence without reason. In Proceedings of the 12th International Joint Conference on Artificial Intelligence, volume I, (pp 569-595). Morgan Kaufmann, San Mateo, CA.

Cablitz, G. (2002). Marquesan. Unpublished dissertation, University of Kiel.

Clancey, W. J. (1993). Situated action: A neuropsychological interpretation response to Vera and Simon. Cognitive Science, 17, 87-116.

Clancey, W. J. (2002). Simulating activities: Relating motives, deliberation, and attentive coordination. Cognitive Systems Research 3(3), 471-499.

Clark, E. V. (1973). Non-linguistic strategies and the acquisition of word meanings.

Cognition, 3, 161-182. 
Dautenhahn, K. / Ogden, B. \& Quick, T. (2002). From embodied to socially embedded agents - implications for interaction-aware robots. Cognitive Systems Research, 3(3), 397-428.

Fischer, K. (2000). What is a situation? In Proceedings of Götalog 2000, $4^{\text {th }}$ Workshop on the Semantics and Pragmatics of Dialogue. Gothenburg Papers in Computational Linguistics 00$5,85-92$.

Franklin, S. \& Graesser, A. (1996). Is it an agent, or just a program? A taxonomy for autonomous agents. In J. P. Müller, M. J. Woolridge, \& N. R. Jennings (Eds.), Intelligent Agents III. Agent Theories, Architectures, and Languages, (pp. 21-35). Springer. Franklin, S. P. (1995). Artificial minds. MIT Press, Cambridge, Mass. Glenberg. A. M. (1997). What memory is for. Behavioural and Brain Sciences, 20(1), 1-55. Goecke, K. U. \& Milde, J.-T. (1998). Situations- und Aktionsbeschreibungen durch einen teilautonomen Montageroboter. In Computers, Linguistics, and Phonetics between Language and Speech. Proceedings of the 4th Conference on Natural Language Processing KONVENS 98, volume 1, (pp. 331-335). Peter Lang, Frankfurt a. M.

Greeno, J. G. / Chi, M. T. H. / Clancey, W. J. \& J. Elman, (Eds.) (1993). Cognitive science special issue: Situated action. Ablex, Norwood, NJ.

Harnad, S. (1990). The symbol grounding problem. Physica D, 42, 335-346.

Herweg, M. (1989). Ansätze zu einer semantischen Beschreibung topologischer Prapositionen. In C. Habel, M. Herweg, \& K. Rehkämper, editors, Raumkonzepte in Verstehensprozessen. Interdisziplinäre Beiträge zu Sprache und Raum, (pp. 99-127). Niemeyer, Tübingen.

Howard, I. P. (1982). Human visual orientation. John Wiley \& Sons, Chichester, New York, Brisbane, Toronto.

Keijzer, F. (2002). Representation in dynamical and embodied cognition. Cognitive Systems Research, 3(3), 275-288. 
Lakoff, G. (1987). Cognitive models and prototype theory. In U. Neisser, (Ed.), Concepts and conceptual development: Ecological and intellectual factors in categorization, (pp. 63-100). Cambridge University Press, Cambridge.

Langacker, R. W. (1987). Foundations of cognitive grammar. Vol. 1: Theoretical prerequisites. Stanford University Press, Stanford.

Levinson, S. C. (1996). Frames of reference and Molyneux's question: Crosslinguistic evidence. In P. Bloom, M. A. Peterson, \& L. Nadel, (Eds.), Language and space, (pp. 109169). MIT Press, Cambridge, Mass.

Madole, K. L. \& Oakes, L. M. (1999). Making sense of infant categorization: Stable processes and changing representations. Developmental Review, 19, 263-296.

Malcolm, C. \& Smithers, T. (1990). Symbol grounding via a hybrid architecture in an autonomous assembly system. Robotics and Autonomous Systems, 6, 123-144.

Margolis, E. \& Laurence, S. (Eds.) (1999). Concepts: core readings. MIT Press, Cambridge. Milde, J.-T. (2000). LOKUTOR: Towards a believable communicative agent. In J. Rickel, W. L. Johnson, \& J. Lester, (Eds.), Achieving human-like behavior in interactive animated agents. Fourth International Conference on Autonomous Agents, 2000.

Milde, J.-T. / Strippgen, S. \& Peters, K. (1997). Situated communication with robots. In First international workshop on human-computer conversation, Bellagio, Italy, July.

Neumann, O. \& Prinz, W. (1990) (Eds.). Relationships between perception and action. Springer.

Nilsson, N. J. (1984). Shakey the robot. Technical report, SRI International. Technical Note 323, Stanford, CA.

Rehm, M. (2001a). Language guiding concept formation in artificial agents. In Holmer, Svantesson, \& Viberg, (Eds.), Proceedings of the 18th Scandinavian Conference of Linguistics, (pp. 241-253). Travaux de l'Institut de Linguistique de Lund. 
Rehm, M. (2001b). LOKATOR - Multimodale Bedeutungskonstitution in situierten Agenten. Bielefeld University, Online publication, URL: http://archiv.ub.unibielefeld.de/disshabi/2001/0073.

Riegler, A. (2002). When is a cognitive system embodied. Cognitive Systems Research, 3(3), $339-348$.

Rohlfing, K.J. (2001). No preposition required. The role of prepositions for the understanding of spatial relations in language acquisition. In S. Niemeyer, M. Pütz, \& R. Dirven, (Eds.), Applied cognitive linguistics I: Theory and language acquisition, (pp. 229-247). Mouton de Gruyter, Berlin.

Rohlfing. K.J. (2002). UNDERstanding. How infants acquire the meaning of UNDER and other spatial relational terms. Bielefeld University, Online publication, URL: http://archiv.ub.uni-bielefeld.de/disshabi/2002/0026/ index.htm. Schyns, P. G. \& Rodet, L. (1997). Categorization creates functional features. Journal of Experimental Psychology: Learning, Memory, and Cognition, 23(3), 681-696.

Semin, G. R. \& Smith, E. R. (2002). Interfaces of social psychology with situated and embodied cognition. Cognitive Systems Research, 3(3), 385-396.

Senft, G. (1997). Introduction. In G. Senft, (Ed.), Referring to space - studies in Austronesian and Papuan languages, (pp. 1-38). Claredon Press, Oxford.

Sinha, C. \& Jensen de Lopez, K. (2000). Language, culture and the embodiment of spatial cognition. Cognitive Linguistics, 11, 17-41.

Sinha, C. (1982). Representational development and the structure of action. In G. Butterworth \& P. Light, (Eds.), Social Cognition: Studies of the Development of Understanding, (pp. 137162). Harvester Press, Brighton, Sussex. 
Sinha, C. (1983). Background knowledge, presupposition, and canonicality. In T. Seiler \& W. Wannenmacher, (Eds.), Concept development and the development of word meaning, (pp. 269-296). Springer, Berlin.

Sinha,C. / Thorseng, L. / Hayashi, M. \& Plunkett, K. (1999). Spatial language acquisition in Danish, English, and Japanese. In P. Broeder \& J. Murre, (Eds.), Language and thought in development. Cross Linguistic Studies, (pp. 95-125). Gunter Narr, Tübingen.

Steels, L. (1993). Building agents out of autonomous behavior systems. In L. Steels \& R. Brooks, (Eds.), The 'artificial life' route to 'artificial intelligence'. Building situated embodied agents (pp. 83-121). Lawrence Erlbaum, Ass. New Haven.

Steels, L. (1996). Perceptually grounded meaning creation. In M. Tokoro, (Ed.), Proceedings of the International Conference on Multi-Agent Systems, (pp. 338-344). AAAI Press.

Thelen, E. \& Smith, L. B. (1994). A dynamic systems approach to the development of cognition and action. MIT Press, Cambridge, Mass.

Thiel, T. (1985): Räumliches Denken und Verständnis von Lokativen beim Spracherwerb. In H. Schweizer (Ed.), Sprache und Raum, (pp. 184-208). Metzler, Stuttgart.

Vygotsky, L. S. (1987). Thinking and speech. In The collected works of L. S. Vygotsky, vol. 1: Problems of general psychology, (pp. 39-285). Plenum Press, New York. 